\title{
Performance Analysis of Admission Control for Integrated Services with Minimum Rate Guarantees
}

\author{
Onno J. Boxma ${ }^{* \dagger}$, Adriana F. Gabor ${ }^{* \dagger}$, Rudesindo Núñez-Queija ${ }^{* \ddagger}$ and Hwee-Pink Tan ${ }^{\dagger}$ \\ *Eindhoven University of Technology, \\ Department of Mathematics and Computer Science, \\ 5600 MB Eindhoven, (The Netherlands)
}

${ }^{\dagger}$ EURANDOM, P.O. Box 513, 5600 MB Eindhoven (The Netherlands)

${ }^{\ddagger}$ CWI, P.O. Box 94079, 1090 GB Amsterdam (The Netherlands)

\begin{abstract}
In this paper, we focus on an admission control strategy for streaming and elastic users that enforces a minimum rate guarantee for each elastic user through pre-emptive capacity reservation. We propose approximations to estimate the performance of this strategy. We apply time-scale decomposition for the limiting regimes, and for non-limiting regimes we propose a novel weighted approximation. Simulation results suggest that the performance is almost insensitive to traffic parameter distributions, and is well estimated by our proposed approximations. Our work is motivated by the integration of services in 3rd generation wireless systems such as UMTS and CDMA 2000.
\end{abstract}

\section{INTRODUCTION}

Future generation broadband networks are expected to support a large variety of applications, typically grouped into two broad categories:

Elastic flows correspond to the transfer of digital documents (e.g., Web pages, emails, stored audio / videos). They are characterized by their size, i.e., the volume of the document to be transferred. These flows are flexible, or "elastic", towards rate fluctuations, with the transfer time as a typical performance measure.

Streaming flows correspond to the real-time transfer of various signals (e.g., voice, streaming audio / video). They are characterized by their duration as well as the transmission rate. For "streaming" applications, stringent transmission rate guarantees are necessary to ensure real-time communication.

Various papers that study the performance of elastic and streaming traffic integration have been published [1], [2], [3], [4], [5], [6], [7]. In terms of resource sharing policy, the classical approach is to give absolute priority to streaming flows (through head-of-line packet marking) in order to offer packet delay and loss guarantees [1], [2], [4]; alternatively, adaptive streaming flows (that are TCP-friendly and mimic elastic flows) are considered in [3], [5], [6]. In terms of modeling approach, while Markovian models have been developed for the exact analysis of the integrated services system, they can be numerically cumbersome. Hence, a fluid model is proposed in [2], [3], [4], [5], [6] to provide closed form results and approximations for limiting traffic regimes based on time scale decomposition.

The above works do not consider the provision of minimum rate guarantees. However, such guarantees are important to maintain satisfactory perceived quality of service, especially for elastic users. Hence, in this paper, we propose such a strategy to enforce minimum rate guarantees through pre-emptive capacity reservation. To model a link that supports such a strategy, we apply both processor sharing and Erlang-loss models and develop approximations for limiting regimes based on timescale decomposition. Comparison with numerical simulations suggests that these approximations are accurate and form performance bounds. This led us to propose a novel weighted approximation for non-limiting regimes.

We describe our model and admission control strategy in detail in Section II. We present the timescale decomposition analysis in Section III and show representative numerical results in Section IV. Some concluding remarks and future directions are 
outlined in Section V. We illustrate the applicability of our model for evaluating the downlink performance of cellular systems in the Appendix.

\section{MODEL}

We consider a link whose limited resource (c Kbps in total) is shared amongst streaming and elastic requests with fixed and minimum rate requirements of $r_{s}$ (Kbps) and $r_{e}$ (Kbps) respectively. We assume that streaming and elastic requests arrive as independent Poisson processes with rate $\lambda_{s}$ and $\lambda_{e}$ respectively. The duration of each admitted streaming request, $d_{s}$, is generally distributed with mean $\frac{1}{\mu_{s}}(\mathrm{sec})$. The corresponding size of each admitted elastic request, $s_{e}$, is generally distributed with mean $f_{e}$ (bits).

A part of the total resource, $c_{s}<c$, is reserved for streaming requests: at any time, $\frac{c_{s}}{r_{s}}$ streaming requests will be guaranteed admission; however, if there are at least $\frac{c_{s}}{r_{s}}$ ongoing streaming requests, then a new streaming request will be admitted as long as the minimum rate $r_{e}$ can be guaranteed for ongoing elastic requests. On the other hand, an elastic request will be admitted as long as ongoing requests can maintain their rate requirements and the number of ongoing elastic requests does not exceed $\frac{c-c_{s}}{r_{e}}$. Note that the capacity unused by streaming requests may be equally shared amongst elastic requests; however, this surplus is immediately re-allocated to streaming requests when a new streaming request arrives.

The above admission control strategy can be quantified as follows: let $\left(N_{s}, N_{e}\right)$ be the number of on-going streaming and elastic requests respectively. Due to the total resource constraint and resource reservation for streaming traffic, $N_{s} r_{s}+$ $N_{e} r_{e} \leq c$ and $N_{e} r_{e} \leq c-c_{s}$. The second inequality ensures that all active elastic flows can be accommodated with their minimum rate by the ensured capacity $c_{e}=c-c_{s}$. Consequently, a new elastic request will be accepted only if $N_{s} r_{s}+\left(N_{e}+1\right) r_{e}$ $\leq c$ and $\left(N_{e}+1\right) r_{e} \leq c-c_{s}$. On the other hand, a new streaming request will be admitted as long as $\left(N_{s}+1\right) r_{s}+N_{e} r_{e} \leq c$. The model is illustrated in Fig. 1.

Note that a different integrated admission control scheme was proposed in [2]. While this scheme aims to ensure equal blocking probabilities, our scheme enforces rate requirements for both types of traffic.

For the convenience of the analysis that follows, we define $K_{e}\left(n_{s}\right)=\left\lfloor\frac{\min \left\{c-n_{s} r_{s}, c_{e}\right\}}{r_{e}}\right\rfloor$ and $K_{s}\left(n_{e}\right)$ $=\left\lfloor\frac{c-n_{e} r_{e}}{r_{s}}\right\rfloor$, where $K_{i}\left(n_{j}\right)$ is the maximum number of type- $i$ flows when $n_{j}$ type- $j$ flows are present.

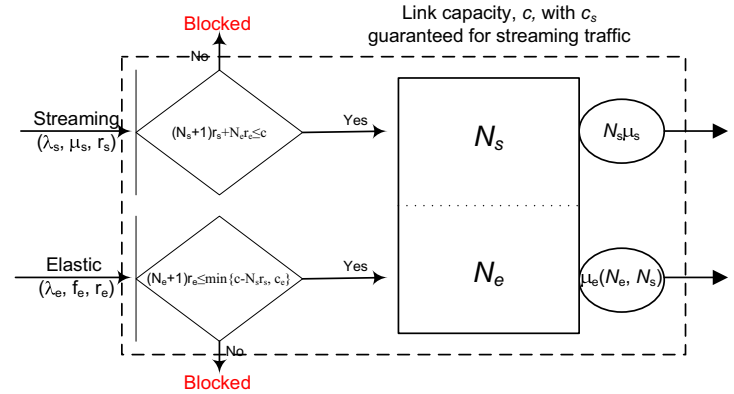

Fig. 1. Model of single link with capacity reservation and minimum rate guarantee for integrated services.

In addition, we denote the conditional probability of event $\mathcal{B}$, given event $\mathcal{A}, P(\mathcal{B} \mid \mathcal{A})$ as $\mathbb{P}_{\mathcal{A}}^{\mathcal{B}}$.

\section{ANALYSIS}

Since exact analysis of our model is nontractable in general and computationally involved when assuming exponentially distributed holding times and file sizes (see [1], [?] for similar models), we develop various approximation techniques and assess their accuracy through comparison with simulation.

\section{A. Quasi-stationary Approximation for Elastic Flows}

For the quasi-stationary approximation, to be denoted $\mathbf{A}(\mathbf{Q})$, we assume that the dynamics of streaming flows take place on a much slower time scale than those of elastic flows. More specifically, we assume that elastic traffic practically reaches statistical equilibrium while the number of active streaming calls remains unchanged. The corresponding condition is that

$$
\mu_{s} E\left[N_{s}\right]+\lambda_{s}<<\frac{c-r_{s} E\left[N_{s}\right]}{f_{e}}+\lambda_{e},
$$

where the expression on the LHS (RHS) corresponds to the average rate at which the number of streaming (elastic) flows changes. Although the above condition cannot be easily checked (due to the dependence on $E\left[N_{s}\right]$ ), it is ensured to be satisfied if $\mu_{s} \frac{c}{r_{s}}+\lambda_{s}<<\lambda_{e}$. This assumption is reasonable when we consider the combination of voice calls (streaming) and web-browsing or email (elastic) applications. Under this assumption, the dynamics of elastic flows can be studied by considering a fixed number of streaming flows, i.e., $N_{s}$ $=n_{s}$. We construct an approximation assuming that the number of active elastic flows instantaneously reaches a new statistical equilibrium whenever the number of streaming flows changes. To avoid any confusion we will mark all quantities (such as 
queue lengths and performance measures) resulting from this approximation approach by adding a superscript ${ }^{Q}$ to the notation.

From the capacity constraint and the reservation policy, it follows that $n_{e} r_{e} \leq \min \{c-$ $\left.n_{s} r_{s}, c_{e}\right\}$. In this case, elastic traffic behaves like an $M / G / 1 / K_{e}\left(n_{s}\right)$ processor-sharing (PS) queue with $K_{e}\left(n_{s}\right)$ service positions, capacity $c-n_{s} r_{s}$ and average departure rate $\mu_{e}\left(n_{s}\right)=\frac{c-n_{s} r_{s}}{f_{e}}$. Hence, from [8],

$$
\begin{aligned}
\mathbb{P}_{N_{s}^{Q}=n_{s}}^{N_{e}^{Q}=n_{e}} & \equiv P\left(N_{e}^{Q}=n_{e} \mid N_{s}^{Q}=n_{s}\right) \\
& =\frac{\rho_{e}\left(n_{s}\right)^{n_{e}}\left(1-\rho_{e}\left(n_{s}\right)\right)}{1-\rho_{e}\left(n_{s}\right)^{K_{e}\left(n_{s}\right)+1}}
\end{aligned}
$$

where $\rho_{e}\left(n_{s}\right)=\frac{\lambda_{e}}{\mu_{e}\left(n_{s}\right)}=\frac{\lambda_{e} f_{e}}{c-n_{s} r_{s}}$. Notice [8] that this expression is insensitive to the file size distribution, other than through its mean. As a further remark, we observe that whether or not $\rho_{e}\left(n_{s}\right)<1$ is of no concern, since $N_{e}^{Q}$ is limited due to the assumption that $r_{e}>0$. Often, when applying a time-scale decomposition, this matter is of importance, giving rise to an additional assumption commonly referred to as uniform stability [4].

Next, we consider the dynamics of streaming flows. When $N_{s}^{Q}=n_{s}$, streaming flows depart at a rate $n_{s} \mu_{s}$. When a new streaming flow arrives, due to admission control, we have two possible scenarios: either the newly arrived streaming flow is accepted or it is blocked. Under our approximation assumptions, the probability of acceptance is $\mathrm{P}\left(N_{e}^{Q} r_{e}+\left(n_{s}+1\right) r_{s} \leq c \mid N_{s}^{Q}=n_{s}\right)$. Notice that the admission probability of streaming flows equals 1 if $\left(n_{s}+1\right) r_{s} \leq c_{s}$. Substituting Eq. (2) into this expression and noting that $N_{e}^{Q} r_{e} \leq c_{e}$, the effective arrival rate of streaming flows, $\Lambda_{s}\left(n_{s}\right)$, is given as follows:

$$
\begin{aligned}
\Lambda_{s}\left(n_{s}\right) & \left.=\lambda_{s} \mathbb{P}_{N_{s}^{Q}=n_{s}}^{N_{e}^{Q}} n_{s}+1\right) \\
& =\lambda_{s} \frac{1-\rho_{e}\left(n_{s}\right)^{K_{e}\left(n_{s}+1\right)+1}}{1-\rho_{e}\left(n_{s}\right)^{K_{e}\left(n_{s}\right)+1}} .
\end{aligned}
$$

Hence, it follows that, for $0 \leq n_{s} \leq\left\lfloor\frac{c}{r_{s}}\right\rfloor$ :

$$
P\left(N_{s}^{Q}=n_{s}\right)=\frac{\prod_{i=0}^{n_{s}-1} \Lambda_{s}(i)}{n_{s} ! \mu_{s}^{n_{s}}} P\left(N_{s}^{Q}=0\right),
$$

where $\mathrm{P}\left(N_{s}^{Q}=0\right)$ can be computed using $\sum_{n_{s}=0}^{\left\lfloor\frac{c}{r_{s}}\right\rfloor} P\left(N_{s}^{Q}=n_{s}\right)=1$. Consequently, it follows that:

$$
P\left(N_{e}^{Q}=n_{e}\right)=\sum_{n_{s}=0}^{\left\lfloor\frac{c}{r_{s}}\right\rfloor} \mathbb{P}_{N_{s}^{Q}=n_{s}}^{N_{e}^{Q}=n_{e}} P\left(N_{s}^{Q}=n_{s}\right) .
$$

In particular, the conditional blocking probability of newly-arrived streaming flows is

$$
P\left(N_{e}^{Q} r_{e}+\left(n_{s}+1\right) r_{s}>c \mid N_{s}^{Q}=n_{s}\right)
$$

. Un-conditioning on $N_{s}^{Q}$, and noting that blocking can occur only for $\left\lfloor\frac{c_{s}}{r_{s}}\right\rfloor \leq n_{s} \leq\left\lfloor\frac{c}{r_{s}}\right\rfloor$, the blocking probability for streaming flows, $p_{s}^{Q}$, is given as follows:

$$
\begin{aligned}
& p_{s}^{Q}=\sum_{n_{s}=\left\lfloor\frac{c_{s}}{r_{s}}\right\rfloor}^{\left\lfloor\frac{c}{r_{s}}\right\rfloor} \mathbb{P}_{N_{s}^{Q}=n_{s}}^{N_{Q}^{Q}>K_{e}\left(n_{s}+1\right)} P\left(N_{s}^{Q}=n_{s}\right) \\
& =1-\frac{1}{\lambda_{s}} \sum_{n_{s}=\left\lfloor\frac{c_{s}}{r_{s}}\right\rfloor}^{\left\lfloor\frac{c}{r_{s}}\right\rfloor} \Lambda_{s}\left(n_{s}\right) P\left(N_{s}^{Q}=n_{s}\right) \text {. }
\end{aligned}
$$

The corresponding blocking probability for elastic flows, $p_{e}^{Q}$, is given as follows:

$$
p_{e}^{Q}=\sum_{n_{s}=0}^{\left\lfloor\frac{c}{r_{s}}\right\rfloor} \mathbb{P}_{N_{s}^{Q}=n_{s}}^{N_{e}^{Q} \geq K_{e}\left(n_{s}\right)} P\left(N_{s}^{Q}=n_{s}\right) .
$$

\section{B. Fluid Approximation for Elastic Flows}

For the fluid approximation, denoted by $\mathbf{A}(\mathbf{F})$, we assume that the dynamics of elastic flows are much slower than those of streaming flows, i.e.,

$$
\frac{c-r_{s} E\left[N_{s}\right]}{f_{e}}+\lambda_{e}<<\mu_{s} E\left[N_{s}\right]+\lambda_{s},
$$

which is certainly true if $\frac{c}{f_{e}}+\lambda_{e}<<\lambda_{s}$. This assumption is valid when we consider the combination of voice calls (streaming) and large file transfer (elastic) applications. Under this assumption, the dynamics of streaming flows can be studied by considering a fixed number of elastic flows. Similar to $\mathbf{A}(\mathbf{Q})$, we will construct an approximating twodimensional process under the assumption that $N_{s}$ immediately reaches steady state, whenever $N_{e}$ changes. This approximation will be reflected in the notation by adding a superscript ${ }^{F}$ whenever not doing so might give rise to confusion.

From the capacity constraint, it follows that $n_{s} r_{s} \leq c-n_{e} r_{e}$. By modeling the streaming flows as an Erlang-loss queue with finite capacity $K_{s}\left(n_{e}\right)$, it follows that:

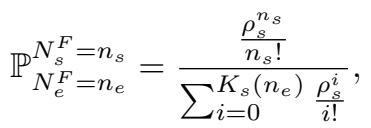

where $\rho_{s}=\frac{\lambda_{s}}{\mu_{s}}$. As before, we emphasize that the above expression depends on the holding time distribution only through its mean.

Next, we consider the dynamics of elastic flows. When $N_{e}^{F}=n_{e}>0$, elastic flows depart at a rate 
$\mu_{e}\left(n_{e}\right)$ given as follows:

$$
\begin{aligned}
\mu_{e}\left(n_{e}\right) & =\frac{E\left[c-N_{s}^{F} r_{s} \mid N_{e}^{F}=n_{e}\right]}{f_{e}} \\
& =\sum_{n_{s}=0}^{K_{s}\left(n_{e}\right)} \frac{c-n_{s} r_{s}}{f_{e}} \mathbb{P}_{N_{e}^{F}=n_{e}}^{N_{s}^{F}=n_{s}}
\end{aligned}
$$

Hence, from the admission control conditions and Eq. (4), the effective arrival rate of elastic flows, $\Lambda_{e}\left(n_{e}\right)$, is given as follows:

$$
\begin{aligned}
\Lambda_{e}\left(n_{e}\right) & =\lambda_{e} \cdot \mathbb{P}_{N_{e}^{F}=n_{e}}^{N_{F}^{F} \leq K_{s}\left(n_{e}+1\right)} \\
& =\lambda_{e} \sum_{l=0}^{K_{s}\left(n_{e}+1\right)} \frac{\frac{\rho_{s}^{l}}{l !}}{\sum_{i=0}^{K_{s}\left(n_{e}\right)} \frac{\rho_{s}^{i}}{i !}} .
\end{aligned}
$$

Using Cohen's results for his generalized PS model [8] for general service times with service rate $\mu_{e}\left(n_{e}\right)$ and arrival rate $\Lambda_{e}\left(n_{e}\right)$, it follows that, for $0 \leq n_{e} \leq\left\lfloor\frac{c_{e}}{r_{e}}\right\rfloor$ :

$$
P\left(N_{e}^{F}=n_{e}\right)=\prod_{i=0}^{n_{e}-1} \frac{\Lambda_{e}(i)}{\mu_{e}(i+1)} P\left(N_{e}^{F}=0\right),
$$

where $\mathrm{P}\left(N_{e}^{F}=0\right) \quad$ can be computed using $\sum_{n_{e}=0}^{K_{s}\left(n_{e}+1\right)} \mathrm{P}\left(N_{e}^{F}=n_{e}\right)=1$. Consequently, $\mathrm{P}\left(N_{s}^{\bar{F}}=n_{s}\right)$ is obtained by substituting Eq. (4) and Eq. (5) into the following expression:

$$
P\left(N_{s}^{F}=n_{s}\right)=\sum_{n_{e}=0}^{\left\lfloor\frac{c_{e}}{r_{e}}\right\rfloor} \mathbb{P}_{N_{e}^{F}=n_{e}}^{N_{s}^{F}=n_{s}} P\left(N_{e}^{F}=n_{e}\right) .
$$

On the other hand, the newly-arrived elastic flow is blocked if $n_{e}=\left\lfloor\frac{c_{e}}{r_{e}}\right\rfloor$ or if $N_{s}^{F} r_{s}+\left(n_{e}+1\right) r_{e}>c$. Hence, the blocking probability for elastic flows, $p_{e}^{F}$, is given by:

$$
\begin{aligned}
p_{e}^{F} & =\sum_{n_{e}=0}^{\left\lfloor\frac{c_{e}}{r_{e}}\right\rfloor-1} \mathbb{P}_{N_{e}^{F}=n_{e}}^{N_{s}^{F}>K_{s}\left(n_{e}+1\right)} P\left(N_{e}^{F}=n_{e}\right) \\
& +P\left(N_{e}^{F}=\left\lfloor\frac{c_{e}}{r_{e}}\right\rfloor\right) .
\end{aligned}
$$

The corresponding blocking probability for streaming flows, $p_{s}^{F}$, is given as follows:

$$
p_{s}^{F}=\sum_{n_{e}=0}^{\left\lfloor\frac{c_{e}}{r_{e}}\right\rfloor} \mathbb{P}_{N_{e}^{F}=n_{e}}^{N_{s}^{F} \geq K_{s}\left(n_{e}\right)} P\left(N_{e}^{F}=n_{e}\right) .
$$

\section{Performance Evaluation}

In this section, we evaluate the performance of an isolated link with elastic and streaming requests (Fig. 1) through simulation for the following parameters: $c=1000 \mathrm{Kbps}, c_{s}=500 \mathrm{Kbps}, r_{s}=60$ Kbps and $r_{e}=40 \mathrm{Kbps}$. We consider the following distributions for $\left(s_{e}, d_{s}\right)$, given that $\mathrm{E}\left[s_{e}\right]=f_{e}$ and $\mathrm{E}\left[d_{s}\right]=\frac{1}{\mu_{s}}$ :

Hyper-exponential distribution : A common distribution used to characterize the behavior of $s_{e}$ is the hyper-exponential distribution with balanced means, which is defined as follows (cf.[9], p. 359): $\forall s \geq 0, P\left(s_{e}>s\right)=\frac{a_{e} e^{\frac{-a_{e} s}{f_{e}}}+e^{\frac{-s}{a_{e} f_{e}}}}{a_{e}+1}$. The parameter $a_{e}$ completely characterizes the behavior of $s_{e}$ and can be interpreted as follows: A fraction $\frac{a_{e}}{a_{e}+1}$ of small elastic requests of mean size $\frac{f_{e}}{a_{e}}$ and a fraction $\frac{1}{a_{e}+1}$ of large elastic requests of mean size $a_{e} f_{e}$. Increasing $a_{e}$ increases the variance of $s_{e}$ and the special case of $a_{e}=1$ corresponds to the exponential distribution.

Erlang distribution : A common distribution to characterize the behavior of $d_{s}$ is the Erlang distribution, which has the following density:

$$
\forall d \geq 0, k>0, f_{s}(d)=\frac{k \mu_{s}\left(k \mu_{s} d\right)^{k-1}}{(k-1) !} e^{-k \mu_{s} d} .
$$

It reduces to an exponential distribution for $k=1$; increasing $k$ reduces the variance for $d_{s}$.

Once the distribution of $\left(d_{s}, s_{e}\right)$ is selected, we characterize each simulation run according to the following procedure:

1. Fix the total offered traffic by choosing the loading factor, $\tau>0$, where $u_{e}+u_{s}=\tau c$, $u_{e}=\lambda_{e} f_{e}$ and $u_{s}=\frac{\lambda_{s} r_{s}}{\mu_{s}}$;

2. For each $\tau$, fix the traffic mix, $\frac{u_{e}}{\tau c}$, by choosing $u_{e}, 0 \leq u_{e} \leq \tau c$;

3. For each traffic mix, select $\left(\lambda_{e}, \lambda_{s}\right)$ to fit one of the following traffic regimes:

a. Quasi-stationary Regime (denoted by $\mathbf{S}(\mathbf{Q})$ ), where Eq. (1) is satisfied;

b. Fluid Regime (denoted by $\mathbf{S}(\mathbf{F})$ ), where Eq. (3) is satisfied;

c. Neutral Regime (denoted by $\mathbf{S}(\mathbf{N})$ ), where neither Eq. (1) nor (3) is satisfied.

We note that in Step 3 of the above procedure, $f_{e}$ and $\mu_{s}$ can be computed once $\left(\tau, \rho_{e}, \lambda_{e}\right.$, $\left.\lambda_{s}\right)$ are specified. The simulation duration, $T$, is selected such that $\min \left\{\lambda_{e}, \lambda_{s}\right\} \cdot T \geq N_{c}$, where $N_{c}$ is chosen (default value $=10000$ ) such that $N_{c} \cdot \min \left\{p_{e}, p_{s}\right\}$ is not too small.

From the simulations, we compute the blocking probability for each type of request. The expected residence time for each admitted elastic request, $E\left[R_{e}\right]$, can be computed in terms of $\left(E\left[N_{e}\right], p_{e}\right)$ using Little's Law as follows:

$$
E\left[R_{e}\right]=\frac{E\left[N_{e}\right]}{\lambda_{e}\left(1-p_{e}\right)} .
$$

We define the stretch, $S_{e}$, by normalizing $E\left[R_{e}\right]$ with $f_{e}$ as follows:

$$
S_{e}=\frac{E\left[R_{e}\right]}{f_{e}} .
$$




\section{A. Performance Insensitivity with Traffic Parameter Distribution}

First, we evaluate the impact of the distribution of $\left(d_{s}, s_{e}\right)$ on the performance of a fully-loaded link $(\tau=1)$ in different traffic regimes. We consider the following traffic mix: (i) $\frac{u_{e}}{c}=0.5$ (Balanced traffic mix) and (ii) $\frac{u_{e}}{c}=0.1$ (Dominant composition of streaming traffic).

For each case, we define 3 sets of simulations as follows: Case I $\left[a_{e}, k\right]=[1,1]$, Case II $\left[a_{e}, k\right]=$ $[100,1]$ and Case III $\left[a_{e}, k\right]=[1,3]$. For each set, we compute the sample mean for $\left(p_{e}, p_{s}, S_{e}\right)$ over all the simulation runs at each traffic mix, and the results are tabulated in Table I. We observe that the performance measures are almost insensitive to the traffic parameter distributions, thus justifying the insensitive approximations proposed here.

\section{B. A Weighted Approximation for Blocking Proba- bilities}

For each scenario in Case I, we plot $\left(p_{e}, p_{s}\right)$ as a function of the traffic mix, $\frac{u_{e}}{c}, 0 \leq u_{e} \leq c$, for each approximation technique in Fig. 2 and Fig. 3 respectively, alongside the corresponding simulation results. Qualitatively, we note that $\mathbf{A}(\mathbf{Q})$ $(\mathbf{A}(\mathbf{F}))$ is accurate in the quasi-stationary (fluid) regime for each metric. The non-monotonicity of the blocking probability for elastic flows is due to the counter-acting effects of reservation and change of traffic composition. Notice that this does not affect the blocking probability for streaming flows.

For the neutral traffic regime, Fig. 2-3 suggest that the blocking probabilities obtained (with simulation) are typically in between $\mathbf{A}(\mathbf{Q})$ and $\mathbf{A}(\mathbf{F})$. Hence, it seems worthwhile to estimate the performance metric $x$ in such a regime by weighing the corresponding metrics obtained with $\mathbf{A}(\mathbf{Q})$ and $\mathbf{A}(\mathbf{F})$ (denoted $\mathbf{A}(\mathbf{W})$ ) as follows:

$x_{\mathbf{A}(\mathbf{W})}=w_{Q} x_{\mathbf{A}(\mathbf{Q})}+\left(1-w_{Q}\right) x_{\mathbf{A}(\mathbf{F})}$, where $w_{Q}$ is the weight allocated to $\mathbf{A}(\mathbf{Q})$ and $x_{\mathbf{A}}$ is the value of $x$ obtained with approximation $\mathbf{A}$.

According to Eq. (1) and Eq. (3), the criteria used to define the traffic regime is the relative dynamics of streaming and elastic flows, given by $\mu_{s} E\left[N_{s}\right]+$ $\lambda_{s}$ and $\frac{c-r_{s} E\left[N_{s}\right]}{f_{e}}+\lambda_{e}$ respectively. Hence, a natural approach to define $w_{Q}$ is as follows:

$$
w_{Q}=\frac{\frac{c-r_{s} E\left[N_{s}\right]}{f_{e}}+\lambda_{e}}{\frac{c-r_{s} E\left[N_{s}\right]}{f_{e}}+\lambda_{e}+\mu_{s} E\left[N_{s}\right]+\lambda_{s}} .
$$

In this way, when the dynamics of elastic flows occur at a faster rate than that of streaming flows (towards quasi-stationary regime), $w_{Q}>0.5$ and vice versa. Accordingly, $E\left[N_{s}\right]=w_{Q} E\left[N_{s}\right]_{\mathbf{A}(\mathbf{Q})}+$ $\left(1-w_{Q}\right) E\left[N_{s}\right]_{\mathbf{A}(\mathbf{F})}$, and together with Eq. (6), $w_{Q}$ can be computed by solving the following quadratic equation:

$$
\text { where } A w_{Q}^{2}+B w_{Q}=C,
$$

$$
\begin{aligned}
A & =\left(E\left[N_{s}\right]_{\mathbf{A}(\mathbf{Q})}-E\left[N_{s}\right]_{\mathbf{A}(\mathbf{F})}\right)\left(\mu_{s} f_{e}-r_{s}\right) \\
B & =c+\left(\lambda_{e}+\lambda_{s}\right) f_{e}+E\left[N_{s}\right]_{\mathbf{A}(\mathbf{F})}\left(\mu_{s} f_{e}-r_{s}\right) \\
& -r_{s}\left(E\left[N_{s}\right]_{\mathbf{A}(\mathbf{F})}-E\left[N_{s}\right]_{\mathbf{A}(\mathbf{Q})}\right) \\
C & =c+\lambda_{e} f_{e}-r_{s} E\left[N_{s}\right]_{\mathbf{A}(\mathbf{F})}
\end{aligned}
$$

We demonstrate the accuracy of $\mathbf{A}(\mathbf{W})$ for the case of a balanced traffic mix. We consider $w_{Q} \in$ $\{0.1,0.2, \cdots, 0.9\}$, and for each $w_{Q}$, we generate simulation runs by selecting 9 sets of traffic parameters. We plot the blocking probabilities obtained alongside the corresponding estimates with $\mathbf{A}(\mathbf{Q})$, $\mathbf{A}(\mathbf{F})$ and $\mathbf{A}(\mathbf{W})$ in Fig. 4. We observe that the blocking probabilities for both types of requests are well-estimated by $\mathbf{A}(\mathbf{W})$.

\section{CONClusions AND Future WORK}

In this study, we evaluate the performance of an admission control strategy that ensures minimum and fixed rates for elastic and streaming requests respectively in an isolated link. We develop approximations to estimate the performance in limiting traffic regimes where the dynamics of both types of requests take place at significantly different time scales. Based on the former, we propose a weighted approximation for non-limiting traffic regimes. Simulation results suggest that the performance is almost insensitive to traffic parameter distributions, and is accurately estimated by the proposed approximations. Our model can, see e.g. [3], be used to assess the performance of downlink communication in $3^{r d}$ generation cellular systems when the difference in distances to the base station within the cell can be neglected. We are currently investigating extensions to include signal attenuation due to path loss. We also plan to investigate more enhanced weighing schemes of the proposed approximations.

\section{APPENDIX}

Let us consider downlink transmissions in UMTS (with W-CDMA in FDD mode) to elastic and streaming users, where the base station can transmit to all active users (denoted by $\mathbb{A}$ ) simultaneously. Let $P$ be the total power available at the base station, and $P_{u}$ be the power transmitted to user $u$, where $P_{u} \leq P$. The power received by a user $u$ is $P_{u}^{r}=P_{u} \Gamma_{u}$, where $\Gamma_{u}$ denotes the attenuation due to path-loss. As a measure of the quality of service for user $u$, we consider the 


\begin{tabular}{|c|c|c|c|c|c|c|c|c|c|c|c|c|c|c|c|c|c|c|}
\hline $\mathrm{u}_{\mathrm{e}} / \mathrm{c}$ & \multicolumn{9}{|c|}{0,5} & \multicolumn{9}{|c|}{0,1} \\
\hline $\mathbf{s}$ & \multicolumn{3}{|c|}{$\mathbf{S}(\mathbf{Q})$} & \multicolumn{3}{|c|}{$\mathbf{S}(\mathbf{F})$} & \multicolumn{3}{|c|}{$\mathrm{S}(\mathrm{N})$} & \multicolumn{3}{|c|}{$\mathbf{S}(\mathbf{Q})$} & \multicolumn{3}{|c|}{$\mathbf{S}(\mathbf{F})$} & \multicolumn{3}{|c|}{$\mathbf{S}(\mathrm{N})$} \\
\hline Case & 1 & II & III & 1 & II & III & 1 & II & III & I & II & III & I & II & IIII & I & II & III \\
\hline$p_{e}$ & 0,086 & 0,086 & 0,085 & 0,057 & 0,055 & 0,058 & 0,069 & 0,069 & 0,071 & 0,122 & 0,119 & 0,120 & 0,075 & 0,072 & 0,072 & 0,103 & 0,101 & 0,110 \\
\hline$p_{s}$ & 0,097 & 0,100 & 0,097 & 0,057 & 0,055 & 0,056 & 0,070 & 0,071 & 0,074 & 0,164 & 0,164 & 0,162 & 0,152 & 0,153 & 0,152 & 0,157 & 0,157 & 0,162 \\
\hline$S_{e}$ & 10,159 & 10,163 & 10,065 & 10,088 & 10,018 & 10,018 & 10,114 & 10,228 & 10,384 & 7,916 & 7,892 & 7,903 & 5,996 & 5,927 & 6,610 & 7,606 & 7,509 & 7,765 \\
\hline
\end{tabular}

TABLE I

IMPACT OF DISTRIBUTION OF TRAFFIC PARAMETERS $\left(s_{e}, d_{s}\right)$ ON $\left(p_{e}, p_{s}, S_{e}\right)$ FOR $\frac{u_{e}}{c}=0.5$ AND 0.1 .
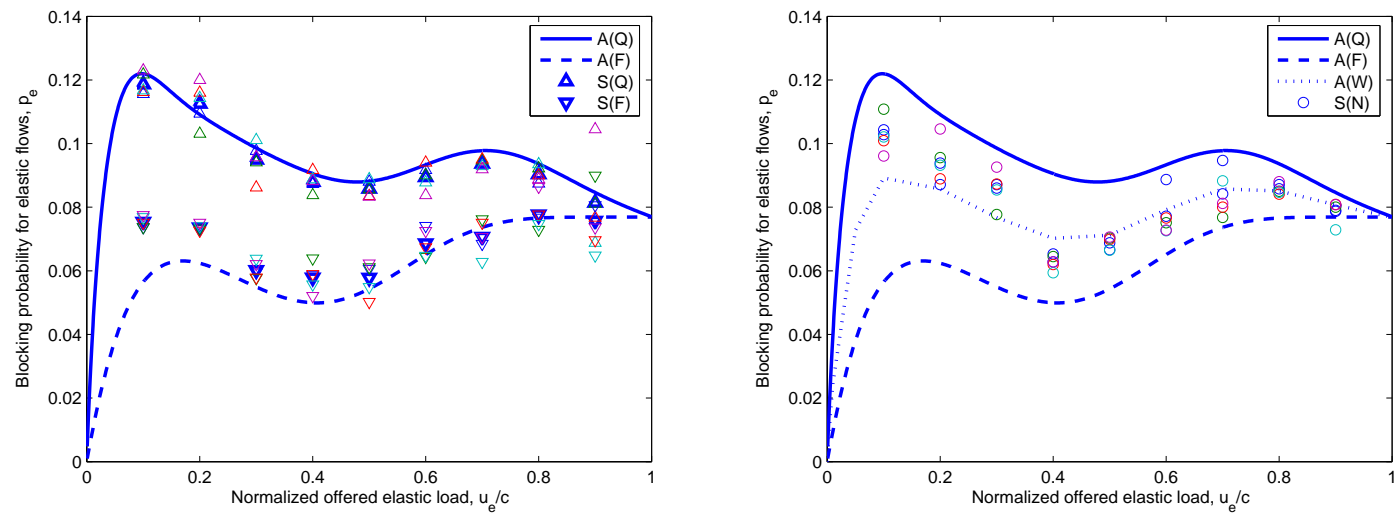

Fig. 2. Blocking probability for elastic requests vs normalized offered elastic load obtained for the 5 cases in quasi-stationary and fluid regimes (left) and neutral regime (right).
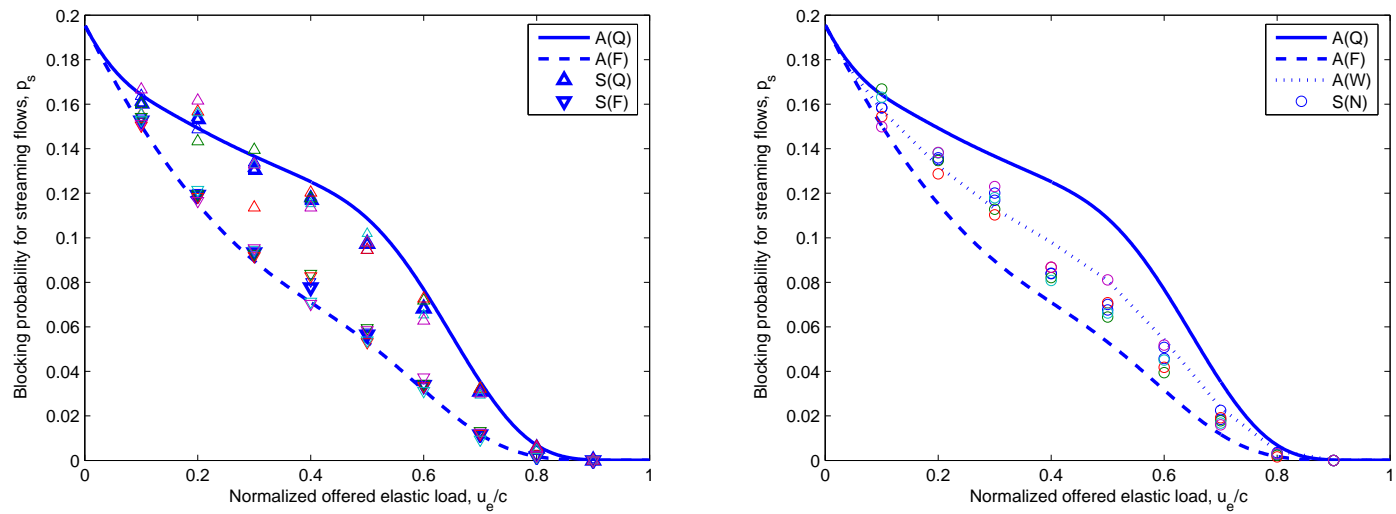

Fig. 3. Blocking probability for streaming requests vs normalized offered elastic load obtained for the 5 cases in quasi-stationary and fluid regimes (left) and neutral regime (right).

energy-per-bit to noise-density ratio, $\left(\frac{E_{b}}{N_{0}}\right)_{u}$, given by

$$
\left(\frac{E_{b}}{N_{0}}\right)_{u}=\frac{W}{R_{u}} \frac{P_{u}^{r}}{\eta+I_{u}^{a}+I_{u}^{r}},
$$

where $W$ is the chip rate, $R_{u}$ is the instantaneous data rate of user $u, \eta$ is the background noise (assumed to be constant throughout the cell) and $\left(I_{u}^{a}, I_{u}^{r}\right)$ is the intra / inter-cell interference at user $u$ respectively. The intra-cell interference arises due to simultaneous transmissions to the other users in the same cell as user $u$, and is given by $I_{r}^{a}=$ $\alpha \sum_{j \in \mathbb{A}, j \neq u} P_{j} \Gamma_{u} \leq \alpha\left(P-P_{u}\right) \Gamma_{u}$, where $\alpha$ is the code non-orthogonality factor. On the other hand, the inter-cell interference is due to the base stations' transmissions in neighboring cells.

To achieve a given target error probability (assumed to be zero here), it is necessary that for each active user $u,\left(\frac{E_{b}}{N_{0}}\right)_{u} \geq \epsilon$, for some threshold 

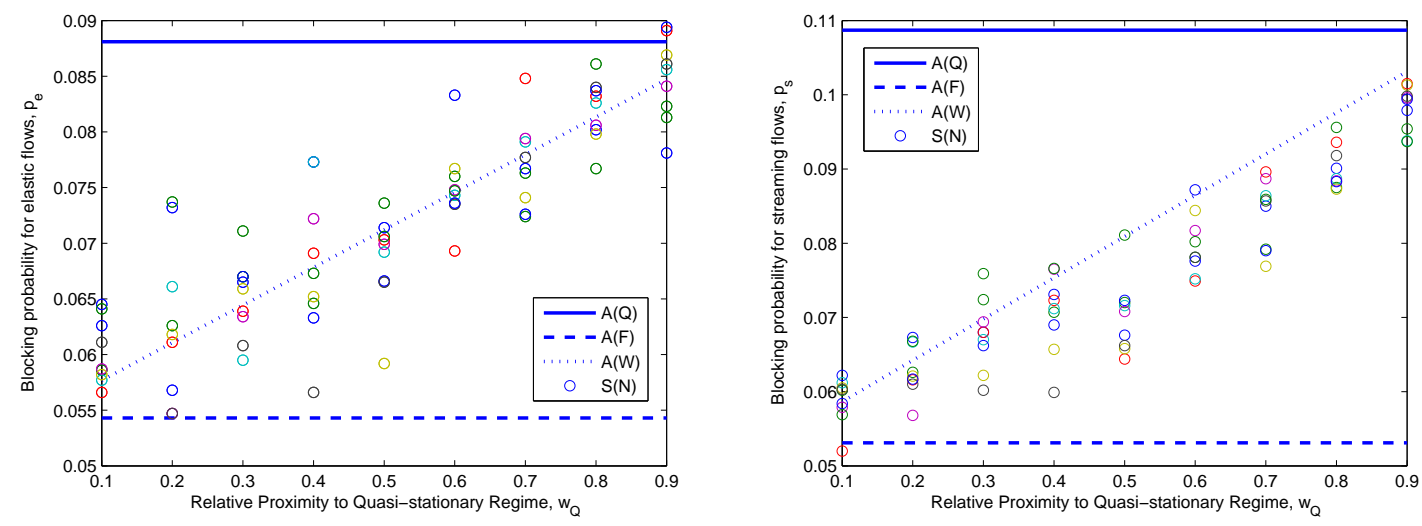

Fig. 4. Blocking probability for elastic (left) and streaming (right) requests for neutral traffic regime, assuming balanced traffic mix, fully loaded cell and exponentially distributed $\left(d_{s}, s_{e}\right)$.

$\epsilon$, which is assumed to be the same for all users. Hence, for each active user $u$, we have the following:

$$
R_{u} \leq \frac{W P_{u}}{\epsilon\left(\alpha\left(P-P_{u}\right)+\frac{\eta+I_{u}^{r}}{\Gamma_{u}}\right)} .
$$

which can be re-written as follows:

$$
\frac{R_{u}}{W+\alpha \epsilon R_{u}} \leq \frac{P_{u}}{\epsilon\left(\frac{\eta+I_{\max }^{r}}{\Gamma_{\min }}+\alpha P\right)} .
$$

Since the function $\frac{R_{u}}{W+\alpha \epsilon R_{u}}$ is an increasing function of $R_{u}$, the following should hold for every elastic request $u$ :

$$
\frac{r_{e}}{W+\alpha \epsilon r_{e}} \leq \frac{P_{u}}{\epsilon\left(\frac{\eta+I_{\max }^{r}}{\Gamma_{\min }}+\alpha P\right)} .
$$

Next, let's assume that a fixed portion of $P, P_{s}$, is reserved for streaming traffic. Then, we have that $\sum_{u \in \mathbb{E}} P_{u} \leq P-P_{s}=P_{e}$.

By summing over the set of $N_{e}$ active elastic users, we obtain the following:

$$
\frac{N_{e} r_{e}}{W+\alpha \epsilon r_{e}} \leq \frac{P_{e}}{\epsilon\left(\frac{\eta+I_{\max }^{r}}{\Gamma_{\min }}+\alpha P\right)} .
$$

Finally, summing Eq. (8) over $\mathbb{A}$, and noting that $R_{u}=r_{s}$ for streaming users, we have:

$$
N_{e} r_{e}+N_{s} r_{s} \leq \frac{P(W+\alpha \epsilon r)}{\epsilon\left(\frac{\eta+I_{\max }^{r}}{\Gamma_{\min }}+\alpha P\right)},
$$

where $r=\max \left(r_{e}, r_{s}\right)$.

Hence, our model can be applied to the downlink transmission scenario in UMTS by defining $c=$ $\frac{P(W+\alpha \epsilon r)}{\epsilon\left(\frac{\eta+I_{\max }^{r}}{\Gamma_{\max }}+\alpha P\right)}$ and choosing $c_{s}=\frac{P_{s}}{P}$. We note that the resulting cell capacity $c$ is conservative, since the admission control criteria is defined based on users at the edge of the cell (where $I_{u}^{r}=I_{\max }^{r}$ and $\left.\Gamma_{u}=\Gamma_{\min }\right)$.

\section{ACKNOWLEDGMENT}

The support of Vodafone is gratefully acknowledged. This research is partially supported by the Dutch Bsik/BRICKS project and is performed within the framework of the European Network of Excellence Euro-NGI.

\section{REFERENCES}

[1] R. Núñez-Queija, J. L. van den Berg, and M. R. H. Mandjes, "Performance evaluation of strategies for integration of elastic and stream traffic," Proc. ITC 16, pp. 1039-1050, 1999. Eds. D. Smith and P. Key. Elsevier, Amsterdam.

[2] N. Benameur, S. B. Fredj, F. Delcoigne, S. OueslatiBoulahia, and J. W. Roberts, "Integrated admission control for streaming and elastic traffic," Lecture Notes in Computer Science, vol. 2156, pp. 69-81, September 2001.

[3] P. Key, L. Massoulié, A. Bain, and F. Kelly, "Fair internet traffic integration: network flow models and analysis," Annales des Telecommunications, vol. 59, pp. 1338-1352, 2004.

[4] F. Delcoigne, A. Proutière, and G. Regnie, "Modeling integration of streaming and data traffic," Performance Evaluation, vol. 55, pp. 185-209, February 2004.

[5] T. Bonald and A. Proutière, "On performance bounds for the integration of elastic and adaptive streaming flows," Proceedings of the ACM SIGMETRICS / Performance, pp. 235-245, June 2004.

[6] P. Key and L. Massoulié, "Fluid Limits and Diffusion Approximations for Integrated Traffic Models," Technical Report MSR-TR-2005-83, Microsoft Research, June 2005.

[7] M. Fischer and T. Harris, "A model for evaluating the performance of an integrated circuit- and packet- switched multiplex structure," IEEE Transactions on Communications, vol. 24, pp. 195-202, February 1976.

[8] J. W. Cohen, "The multiple phase service network with generalized processor sharing," Acta Informatica, vol. 12, pp. 245-284, 1979.

[9] H. C. Tijms, Stochastic Models - An Algorithmic Approach. John-Wiley and Sons, 1994. 